\title{
La forma diferencial de las ecuaciones básicas del flujo unidimensional
}

\author{
G. Sotelo-Ávila y A. Cafaggi-Félix \\ División de Ingeniería Civil, Topográfica y Geodésica \\ Facultad de Ingeniería, UNAM \\ E-mail: soteloa@servidor.unam.mxyacafaggi@servidor.unam.mx
}

(recibido: abril de 2001; aceptado: mayo de 2001)

\begin{abstract}
Resumen
Cuando se plantean las ecuaciones diferenciales del flujo unidimensional es común utilizar sólo los principios básicos de continuidad y la segunda ley de Newton, sin emplear el de energía, ya que este último encuentra aplicación solamente en la ecuación dinámica del flujo gradualmente variado en canales. Este artículo presenta el desarrollo de dichas ecuaciones usando los tres principios, con el propósito de mostrar las diferencias o afinidades entre ecuaciones de energía y cantidad de movimiento, así como las razones para preferir una u otra según sea el caso.
\end{abstract}

Descriptores: flujo unidimensional, ecuaciones básicas en la hidráulica.

Abstract

It is common to use the basic principles of continuity and the second law of Newton, when the diferential equations of one-dimensional flow are derived. This paper shows the derivation of such equations using the three basic principles, with the purpose to show the differences and affinities between the energy equation and the momentum equation and the reasons to prefer some of them in each case.

Keywords: one-dimensional flow, hydraulic basic equations

\section{Antecedentes}

La aplicación de los principios básicos de continuidad, energía y cantidad de movimiento (segunda ley de Newton) a un volumen de control, constituye los métodos de análisis en la hidráulica. El método más utilizado consiste en elegir un volumen de control de tamaño finito, en cuyo caso, el análisis se conoce como integral, ya que la expresión matemática de los principios básicos es con base en integrales de área y de volumen. Éstas se pueden resolver fácilmente si el flujo es permanente; no así cuando el flujo no lo es.

Cuando se elige un volumen de control de tamaño diferencial que encierra a un punto de un campo de flujo tridimensional, el análisis se conoce como diferencial ya que se obtiene un sistema de ecuaciones diferenciales, comúnmente en derivadas parciales, como expresión matemática de solamente dos de los principios básicos: continuidad y cantidad de movimiento; el de energía no se emplea. Es el caso de las conocidas ecuaciones de continuidad y de Navier-Stokes, consideradas como las más generales para cualquier tipo de flujo. La solución directa de dichas ecuaciones se restringe a casos sencillos, por la dificultad de la integración y por la necesidad de conocer e interpretar las condiciones de frontera que deben usarse en cada caso. Es también común utilizar la ecuación sobre uno de los ejes para representar con ella al flujo unidimensional, ignorando con esto que el eje en el primer caso es recto, pero en el segundo puede ser curvo.

El análisis diferencial del flujo unidimensional es el método intermedio, ya que emplea un volumen de control limitado por las fronteras de la conducción y por secciones perpendiculares al flujo, separadas una distancia diferencial en donde se considera la media espacial de las variables en el centroide de cada sección. Es común que se apliquen únicamente dos de los principios básicos: continuidad y cantidad de movimiento y su expresión matemática se convierte en dos ecuaciones diferenciales que contienen los valores medios de las variables, en función de la coordenada que sigue el eje del conducto y del tiempo. Es el caso de las 
DOI: http://dx.doi.org/10.22201/fi.25940732e.2001.02n3.013

ecuaciones del golpe de ariete y del tránsito de ondas de avenidas en almacenamientos y en canales. Sin embargo, en flujos como el permanente gradualmente variado en canales, se prefiere usar el principio de la energía, sin justificar dicha preferencia. Se piensa además que dicho principio y el de la cantidad de movimiento describen cualquier flujo con grados idénticos de precisión, lo cual sólo se cumple cuando se hacen simplificaciones importantes en ambas ecuaciones.

El propósito de este trabajo es presentar el desarrollo de las ecuaciones diferenciales del flujo unidimensional, como expresión matemática de los tres principios fundamentales, así como presentar sus afinidades y diferencias con la idea de generalizar un método de análisis de gran trascendencia en hidráulica.

En todas las aplicaciones se empleará el volumen de control VC deformable, mostrado en la figura 1, limitado lateralmente por la superficie de control SC coincidente con la pared del conducto o con una superficie libre (si existe), además de dos secciones transversales al flujo, separadas la distancia ds, donde $s$ representa la coordenada curvilínea que sigue el eje de la conducción. La otra variable independiente es el tiempo $t$. En cualquier instante el volumen de líquido contenido en el VCes $d v=A d s$, donde $\mathrm{A}$ es función de $(s, t)$ y $d s$ es independiente de ambos.

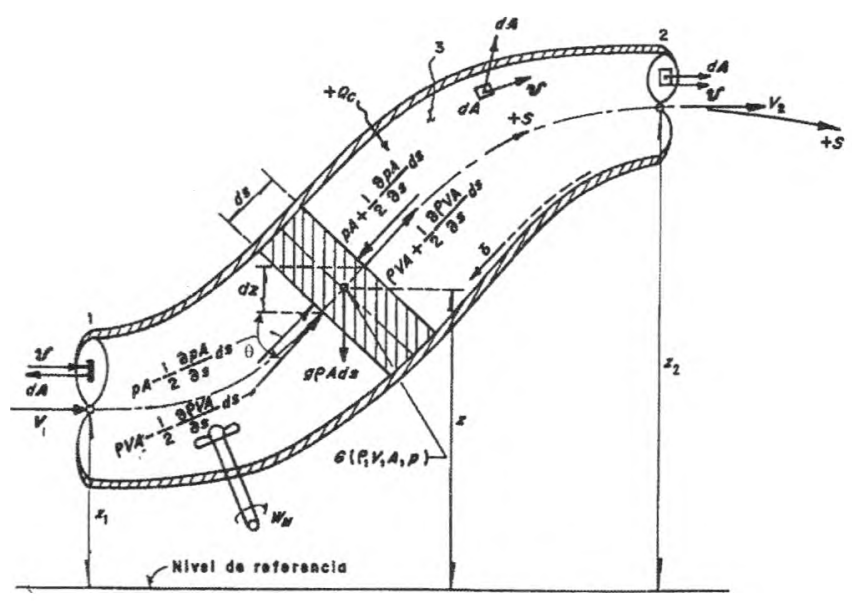

Figura 1. Volumen de control de tamaño diferencial en un flujo unidimensional

\section{La ecuación de continuidad}

La ecuación de transporte de Reynolds permite la expresión matemática del principio de continuidad aplicado a cualquier $\mathrm{VC}$ que es

$$
\oiint_{S C} \rho \mathbf{v} \cdot d A+\frac{\partial}{\partial t} \oiiint_{V C} \rho d v=0
$$

Para el VC elegido, el flujo neto de masa a través de las secciones de flujo es la diferencia entre la que sale y la que entra, es decir:

$$
\frac{\partial(\rho V A)}{\partial s} d s
$$

La rapidez con que varía la masa dentro del VC es: $\partial(\rho \mathrm{A} d s) / \partial t$. Por tanto, el principio establece

$$
\frac{\partial(\rho V A)}{\partial s}+\frac{\partial(\rho A)}{\partial t}=0
$$

Siendo $\rho, V$, A funciones de $s$ y $t$, al desarrollar las derivadas parciales se obtiene

$$
\rho A \frac{\partial V}{\partial s}+\rho V \frac{\partial A}{\partial s}+V A \frac{\partial \rho}{\partial s}+\rho \frac{\partial A}{\partial t}+A \frac{\partial \rho}{\partial t}=0
$$

Al dividir entre $\rho$ A y ordenar términos, resulta

$$
\frac{\partial V}{\partial s}+\frac{1}{A}\left(\frac{\partial A}{\partial s} V+\frac{\partial A}{\partial t}\right)+\frac{1}{\rho}\left(\frac{\partial \rho}{\partial s} V+\frac{\partial \rho}{\partial t}\right)=0
$$

Con $V=d s / d t$ y por el desarrollo de la derivada total, también se obtiene

$$
\frac{\partial V}{\partial s}+\frac{1}{A} \frac{d A}{d t}+\frac{1}{\rho} \frac{d \rho}{d t}=0
$$

Las ecuaciones $1 \mathrm{a}, 1 \mathrm{~b}$ y $1 \mathrm{c}$ son expresiones generales equivalentes de la ecuación diferencial de continuidad, las cuales adoptan alguna forma particular según el tipo de flujo que se analice, como se presenta a continuación.

a) Flujo no permanente en conductos a presión (golpe de ariete)

De la definición de módulo de compresibilidad, $K=d p / d \rho / \rho$, se puede escribir

$$
\frac{1}{\rho} \frac{d \rho}{d t}=\frac{1}{K} \frac{d p}{d t}
$$

El segundo término de la ecuación 1c se debe a la elasticidad de las paredes del tubo y a su rapidez de deformación con la presión. Si se considera un conducto de sección transversal circular, la rapidez con que cambia la fuerza de tensión por unidad de longitud (figura 2 ) está dada por $(D / 2) d p / d t$; donde $D$ es el diámetro del conducto. Si esta cantidad se divide entre el espesor de la pared del conducto 
$e$, se obtiene la rapidez con que cambia el esfuerzo unitario, $\left(D / 2_{e}\right) d p / d t$; si a su vez, esta cantidad se divide entre el módulo de elasticidad de Young para el material del tubo $E_{t}$, resulta la rapidez de cambio de la deformación unitaria y si además se multiplica por el radio de la tubería $D / 2$, se obtiene la rapidez de expansión radial. Finalmente, al multiplicar por el perímetro $\pi D$, resulta la rapidez de crecimiento del área transversal del tubo:

$$
\frac{d A}{d t}=\frac{D}{2 e E_{\imath}} \frac{d p}{d t} \frac{D}{2} \pi D
$$

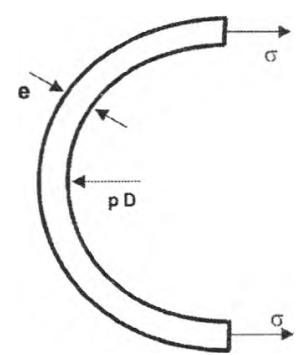

Figura 2. Fuerza de tensión en la pared de un tubo

Sustituyendo las ecuaciones 2 y 3 en la $1 \mathrm{c}$

$$
\frac{1}{K} \frac{d p}{d t}\left(1+\frac{K}{E_{t}} \frac{D}{e}\right)+\frac{\partial V}{\partial s}=0
$$

haciendo

$$
a=\sqrt{\frac{K / \rho}{1+\left(K / E_{t}\right)(D / e)}}
$$

donde $a$ es la celeridad de la onda de presión y toma en cuenta las propiedades del tubo y del conducto. Al sustituir la ecuación 5 en la 4 se tiene

$$
\frac{1}{\rho} \frac{d p}{d t}+a^{2} \frac{\partial V}{\partial s}=0
$$

por otro lado, como $p=\rho g(H-z)$, donde $H$ es la carga piezométrica, se puede escribir

$$
\frac{d p}{d t}=V \frac{\partial p}{\partial s}+\frac{\partial p}{\partial t}=V g \rho\left(\frac{\partial H}{\partial s}-\frac{\partial z}{\partial s}\right)+g \rho\left(\frac{\partial H}{\partial t}-\frac{\partial z}{\partial t}\right)
$$

el cambio de $\rho$ con respecto a $s$ o a $t$ es mucho menor que el cambio de $H$ con respecto a $s$ a $t$, por ello $\rho$ se considera constante. Como el tubo se encuentra en reposo, $\partial z / \partial t=0 \mathrm{y}$ se define $\partial z / \partial s=\operatorname{sen} \theta ;$ si se sustituye la ecuación 7 en la 6 y este resultado en la ecuación $1 \mathrm{c}$; se tiene

$$
\frac{a^{2}}{g} \frac{\partial V}{\partial s}+V \frac{\partial H}{\partial s}+\frac{\partial H}{\partial t}+V \operatorname{sen} \theta=0
$$

expresión que constituye la ecuación de continuidad para un líquido compresible en una tubería elástica.

Flujo no permanente en cámaras de oscilación

Una cámara de oscilación es un tubo vertical conectado a la tubería de presión. En este análisis se considera que el flujo en el conducto es incompresible y que las paredes son rígidas. Con referencia en la figura 3, cuando se presenta oscilación en la cámara, la velocidad en esta es $V_{c}=d z / d t$, y el gasto en el mismo es $Q_{c}=V_{c} A_{c}$, y de la ecuación $1 \mathrm{a}$, con $\partial \rho \mathrm{A} / \partial \mathrm{t}=0$, se tiene

$$
V_{t} A_{t}=\frac{d z}{d t} A_{c}+Q_{T}
$$

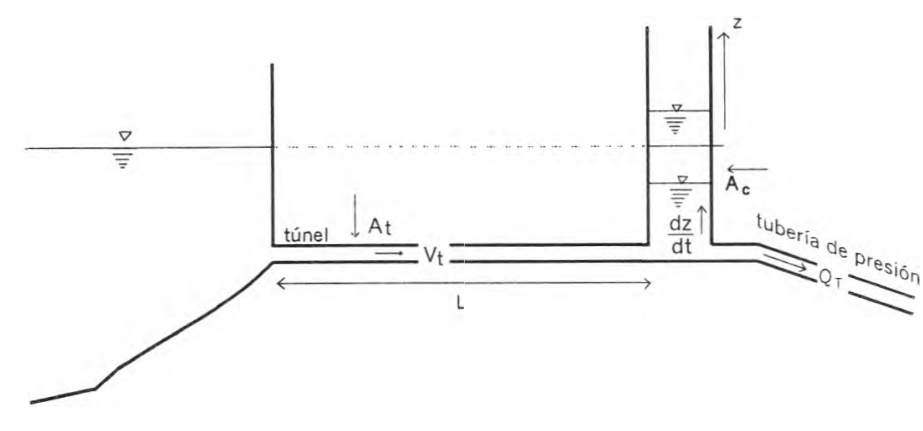

Figura 3. Cámara de oscilación

b) Flujo no permanente en canales (ondas de avenida)

En este caso, $\rho$ es constante y la ecuación $1 \mathrm{~b}$ se simplifica a la forma

$$
\frac{\partial V}{\partial s}+\frac{V}{A} \frac{\partial A}{\partial s}+\frac{1}{A} \frac{\partial A}{\partial t}=0
$$

c) Flujo permanente en canales con gasto variable ( $\rho$ es constante)

De la ecuación 1a

$$
\frac{\partial V A}{\partial s}=\frac{\partial Q}{\partial s}=V \frac{\partial A}{\partial s}+A \frac{\partial V}{\partial s}
$$


o bien, con $\mathrm{q}^{*}=\partial \mathrm{Q} / \partial \mathrm{s}$

$$
q^{*}=V \frac{\partial A}{\partial s}+A \frac{\partial V}{\partial s}
$$

que es la ecuación de continuidad del flujo espacialmente variado.

d) Flujo permanente en canales con gasto constante

El segundo término de la ecuación 1 a desaparece y $\rho$ es constante; por tanto, resulta $\partial \mathrm{VA} / \partial \mathrm{s}=0 \mathrm{y} \mathrm{Q}=\mathrm{VA}=$ constante.

\section{La ecuación de la energía}

El principio de la energía establece que

$$
\begin{gathered}
\oiint_{S C}\left(u+g z+\frac{v^{2}}{2}\right) \rho v \cdot d \mathrm{~A}+\frac{\partial}{\partial t} \oiiint_{v c}\left(u+g z+\frac{v^{2}}{2}\right) \rho d v+ \\
\oiint_{s c} p v \cdot d \mathrm{~A}-P_{M}-\widetilde{Q}_{C}=0
\end{gathered}
$$

O bien, reordenando términos resulta

$$
\begin{gathered}
\oiint_{S C}\left(g z+\frac{p}{\rho}+\frac{v^{2}}{2}\right) \rho v \cdot d A+\frac{\partial}{\partial t} \oiiint_{v C}\left(g z+\frac{v^{2}}{2}\right) \rho d v- \\
P_{M}+\oiint_{S C} u \rho v \cdot d A+\frac{\partial}{\partial t} \oiiint_{v C} u \rho d v-\widetilde{Q}_{C}=0
\end{gathered}
$$

Los tres últimos términos representan la irreversibilidad del proceso en el flujo, es decir, el flujo neto de energía interna $u$ (por unidad de masa) más la rapidez del cambio de energía interna dentro del VC, más el intercambio de calor con el exterior en la unidad de tiempo $\widetilde{Q}_{\mathrm{C}}$.

Los restantes términos son los comúnmente empleados para las variables, además es común eliminar el término de potencia de flecha, $\mathrm{P}_{\mathrm{M}}$, debido al trabajo efectuado por alguna máquina desde el exterior.

La ecuación equivalente a la 13 para el VC de la figura 1 es

$$
\begin{gathered}
\frac{\partial}{\partial s}\left[\left(g z+\frac{p}{\rho}\right) \rho V A+\iint_{A} \frac{v^{2}}{2} \rho v d A\right] d s+ \\
\frac{\partial}{\partial t}\left[\left(g z+\frac{1}{A} \iint_{A} \frac{v^{2}}{2} d A\right) \rho A d s\right]+\frac{\partial(u \rho V A)}{\partial s} d s+ \\
\frac{\partial}{\partial t}(u \rho A d s)-\widetilde{Q}_{C}=0
\end{gathered}
$$

donde se han utilizado los valores medios de las variables en los términos lineales; no así en los cuadráticos, los que hay que calcular en la forma acostumbrada:

$$
\iint_{A} \frac{v^{2}}{2} \rho v d A=\frac{\rho}{2} \iint_{A} v^{3} d A=\frac{V^{2}}{2} \rho V A \frac{1}{A} \iint_{A}\left(\frac{v}{V}\right)^{3} d A=\alpha \frac{V^{2}}{2} \rho Q(15)
$$

donde se ha sustituido a $Q=V A$ y también

$$
\alpha=\frac{1}{A} \iint_{A}\left(\frac{v}{V}\right)^{3} d A
$$

que es el coeficiente de Coriolis para corregir el cálculo del flujo de energía cinética efectuado con la velocidad media $V$.

En la misma forma

$$
\frac{1}{A} \iint_{A} \frac{v^{2}}{2} d A=\frac{v^{2}}{2} \frac{1}{A} \iint_{A}\left(\frac{v}{V}\right)^{2} d A=\frac{v^{2}}{2} \beta=\frac{1}{2} \frac{Q}{A} \beta V
$$

donde

$$
\beta=\frac{1}{A} \iint_{A}\left(\frac{v}{V}\right)^{2} d A
$$

es el coeficiente de Boussinesq para corregir el cálculo de la cantidad de movimiento empleando la velocidad media.

Por tanto, la ecuación 14 se convierte en

$$
\begin{gathered}
\frac{\partial}{\partial s}\left[\left(g z+\frac{p}{\rho}+\alpha \frac{V^{2}}{2}\right) \rho Q\right]+\frac{\partial}{\partial t}\left[\left(g z+\frac{1}{2} \frac{Q}{A} \beta V\right) \rho A\right] \\
+\frac{\partial u \rho Q}{\partial s}+\frac{\partial u \rho A}{\partial t}-\frac{\widetilde{Q}_{C}}{d s}=0
\end{gathered}
$$

Algunos de los términos se pueden desarrollar como sigue:

$$
\frac{\partial}{\partial t}(g z \rho \mathrm{A})=g \rho \mathrm{A} \frac{\partial z}{\partial t}+g z \frac{\partial \rho \mathrm{A}}{\partial t}=g z \frac{\partial \rho \mathrm{A}}{\partial t}
$$

ya que $\partial z / \partial t=0$, puesto que la geometría del eje del conducto no depende del tiempo, es decir, permanece estático. Otro término es

$$
\begin{gathered}
\frac{1}{2} \frac{\partial}{\partial t}(\rho Q \beta V)=\frac{1}{2} \rho Q \frac{\partial \beta V}{\partial t}+\frac{1}{2} \beta V \frac{\partial \rho A V}{\partial t} \\
=\frac{1}{2} \rho Q \frac{\partial \beta V}{\partial t}+\frac{1}{2} \beta V\left(\rho A \frac{\partial V}{\partial t}+V \frac{\partial \rho A}{\partial t}\right) \\
=\frac{1}{2} \rho Q\left(\beta \frac{\partial V}{\partial t}+V \frac{\partial \beta}{\partial t}\right)+\frac{1}{2} \rho Q \beta \frac{\partial V}{\partial t}+\frac{1}{2} \beta V^{2} \frac{\partial \rho A}{\partial t} \\
\frac{1}{2} \frac{\partial}{\partial t}(\rho, Q \beta V)=\rho Q \beta \frac{\partial V}{\partial t}+\beta \frac{V^{2}}{2} \frac{\partial \rho A}{\partial t}
\end{gathered}
$$


donde se ha considerado que $\partial \beta / \partial t=0$. Por último, se tiene el término

$$
\begin{aligned}
\frac{\partial u \rho Q}{\partial s} & +\frac{\partial u \rho A}{\partial t}=u \frac{\partial \rho Q}{\partial s}+\rho Q \frac{\partial u}{\partial s}+u \frac{\partial \rho A}{\partial t}+\rho A \frac{\partial u}{\partial t} \\
& =u\left(\frac{\partial \rho Q}{\partial s}+\frac{\partial \rho A}{\partial t}\right)+\rho A\left(\frac{\partial u}{\partial s} V+\frac{\partial u}{\partial t}\right)
\end{aligned}
$$

Según la ecuación de continuidad 1a, el primer paréntesis de la ecuación anterior vale cero.

Con $V=d s / d t$, el segundo paréntesis se desarrolla como sigue:

$$
\frac{\partial u}{\partial s} V+\frac{\partial u}{\partial t}=\frac{\partial u}{\partial s} \frac{d s}{d t}+\frac{\partial u}{\partial t}=\frac{d u}{d t}=\frac{d u}{d s} \frac{d s}{d t}=V \frac{d u}{d s}
$$

Por tanto, resulta

$$
\frac{\partial u \rho Q}{\partial s}+\frac{\partial u \rho A}{\partial t}=\rho Q \frac{d u}{d s}
$$

Se sustituyen las ecuaciones 20 a 22 en la 19 y al agrupar términos se obtiene

$$
\begin{aligned}
& \frac{\partial}{\partial s}\left[\left(z+\frac{p}{g \rho}+\alpha \frac{V^{2}}{2 g}\right) g \rho Q\right]+\left(z+\beta \frac{V^{2}}{2 g}\right) g \frac{\partial \rho A}{\partial t} \\
& +\rho, Q \beta \frac{\partial V}{\partial t}+\rho Q \frac{d u}{d s}-\frac{\widetilde{Q}_{C}}{d s}=0
\end{aligned}
$$

O bien

$$
\begin{gathered}
g \rho Q \frac{\partial}{\partial s}\left(z+\frac{p}{g \rho}+\alpha \frac{V^{2}}{2 g}\right)+\left(z+\frac{p}{g \rho}+\alpha \frac{V^{2}}{2 g}\right) g \frac{\partial \rho Q}{\partial s}+ \\
\left(z+\beta \frac{V^{2}}{2 g}\right) g \frac{\partial \rho A}{\partial t}+\rho Q \beta \frac{\partial V}{\partial t}+\rho Q \frac{d u}{d s}-\frac{\widetilde{Q}_{C}}{d s}=0
\end{gathered}
$$

De la ecuación de continuidad 1a, $\partial(\rho \mathrm{Q}) / \partial \mathrm{s}=-\partial(\rho \mathrm{A}) / \partial \mathrm{t}$, por tanto, la ecuación diferencial se convierte en

$$
\begin{gathered}
g \rho Q \frac{\partial}{\partial s}\left(z+\frac{p}{g \rho}+\alpha \frac{V^{2}}{2 g}\right)-\left(z+\frac{p}{g \rho}+\alpha \frac{V^{2}}{2 g}-z-\beta \frac{V^{2}}{2 g}\right) g \frac{\partial \rho A}{\partial t} \\
+\rho Q \beta \frac{\partial V}{\partial t}+\rho Q \frac{d u}{d s}-\frac{\widetilde{Q}_{C}}{d s}=0
\end{gathered}
$$

Al reducir y dividir entre $g \rho Q$, resulta

$$
\frac{\partial}{\partial s}\left(z+\frac{p}{g \rho}+\alpha \frac{V^{2}}{2 g}\right)-\left[\frac{p}{g \rho}+(\alpha-\beta) \frac{V^{2}}{2 g}\right\rceil \frac{1}{\rho Q} \frac{\partial \rho A}{\partial t}
$$

$$
+\frac{\beta}{g} \frac{\partial V}{\partial t}+\frac{1}{g} \frac{d u}{d s}-\frac{\widetilde{Q}_{C}}{g \rho Q d s}=0
$$

Es posible el siguiente desarrollo

$$
\frac{1}{\rho Q} \frac{\partial \rho A}{\partial t}=\frac{1}{\rho Q Q}\left(\rho \frac{\partial A}{\partial t}+A \frac{\partial \rho}{\partial t}\right)=\frac{1}{V}\left(\frac{1}{A} \frac{\partial A}{\partial t}+\frac{1}{\rho} \frac{\partial \rho}{\partial t}\right)=\frac{1}{Q} \frac{\partial A}{\partial t}
$$

donde se ha aceptado que $(1 / A)(\partial A / \partial t)>>(1 / \rho)(\partial \rho / \partial t)$, aun en golpe de ariete.

Además, la irreversibilidad del proceso se representa en la forma

$$
\frac{d I_{r}}{d s}=\frac{1}{g} \frac{d u}{d s}-\frac{\widetilde{Q}_{C}}{g \rho, Q d s}
$$

donde el primer término es el cambio de energía interna por unidad de distancia y por unidad de peso; el segundo, es la rapidez de disipación de calor (también por la unidad de distancia y por unidad de peso). La irreversibilidad se interpreta en hidráulica como una pérdida de la energía total a lo largo del conducto en cada instante, es decir

$$
\frac{d I_{r}}{d s}=\frac{d h_{r}}{d s}=S_{E}
$$

donde $S_{E}$ es la pendiente de la línea de energía.

La ecuación 23 se convierte en

$$
\begin{gathered}
\frac{\partial}{\partial s}\left(z+\frac{p}{g \rho}+\alpha \frac{V^{2}}{2 g}\right)-\left[\frac{p}{g \rho}+(\alpha-\beta) \frac{V^{2}}{2 g}\right] \\
\frac{1}{Q} \frac{\partial A}{\partial t}+\frac{\beta}{g} \frac{\partial V}{\partial t}+\frac{d h_{r}}{d s}=0
\end{gathered}
$$

que es la forma general de la ecuación de la energía para el flujo unidimensional no permanente y compresible de un líquido. Adquiere formas distintas según el tipo de flujo al que se aplique, como se presenta a continuación.

a) Cuando sólo se acepta que $\alpha=\beta=1$, la ecuación se simplifica y resulta

$$
\frac{\partial}{\partial s}\left(z+\frac{p}{g \rho}+\frac{V^{2}}{2 g}\right)-\frac{p}{g \rho} \frac{1}{Q} \frac{\partial A}{\partial t}+\frac{1}{g} \frac{\partial V}{\partial t}+\frac{d h_{r}}{d s}=0
$$

b) Cuando el flujo es no permanente en un conducto cerrado rígido sin cambios violentos de la presión (oscilaciones en masa) $\partial \mathrm{A} / \partial \mathrm{t}=0$, y la ecuación 26 se reduce a la forma

$$
\frac{\partial}{\partial s}\left(z+\frac{p}{g \rho}+\alpha \frac{V^{2}}{2 g}\right)+\frac{\beta}{g} \frac{\partial V}{\partial t}+\frac{d h_{r}}{d s}=0
$$


DOI: http://dx.doi.org/10.22201/fi.25940732e.2001.02n3.013

donde puede admitirse que $\alpha \cong \beta \cong 1$. Si se trata de un canal, subsiste la ecuación 26, o la 27 en su caso (siempre que la sección no sea compuesta).

c) Cuando el flujo es permanente, se anulan los términos que dependen del tiempo y $d h_{\mathrm{r}} / d s=\partial h_{r} / \partial \mathrm{s}$, por tanto, la ecuación 26 se simplifica y se integra fácilmente entre las secciones 1 y 2 de la figura 1 , como sigue:

$$
\begin{gathered}
\frac{\partial}{\partial s}\left(z+\frac{p}{g \rho}+\alpha \frac{V^{2}}{2 g}+h_{r}\right)=0 \\
z_{1}+\frac{p_{1}}{g \rho}+\alpha_{1} \frac{V_{1}^{2}}{2 g}=z_{2}+\frac{p_{2}}{g \rho}+\alpha_{2} \frac{V_{2}^{2}}{2 g}+h_{r}
\end{gathered}
$$

donde $h_{r}$, es la pérdida total de energía de 1 a 2 .

A la ecuación 30 se puede agregar el término $H_{M}=P_{M} / g \rho Q$, energía de flecha por unidad de peso. Cuando se elimina $h_{\mathrm{r}}$ y se hace $\alpha_{1}=\alpha_{2}=1$ se concluye la conocida ecuación de Bernoulli para la línea de corriente que coincide con el eje.

\section{La ecuación de la cantidad de movimiento}

La segunda ley de Newton aplicada a un VC cualquiera se expresa en la forma

$$
F_{p}+F_{\tau}+F_{c}=\oiint_{s c} v \rho(v \cdot d A)+\frac{\partial}{\partial t} \oiiint_{v c} v \rho d A
$$

Cuando se aplica al VC de la figura 1 es necesario obtener cada una de las fuerzas. La resultante $F_{p}$ de las fuerzas debidas a la presión en las dos secciones a la distancias, más la que ocurre por el incremento de área, es

$$
\begin{gathered}
\left(p A-\frac{1}{2} \frac{\partial p A}{\partial s} d s\right)-\left(p A+\frac{1}{2} \frac{\partial p A}{\partial s} d s\right)+p \frac{\partial A}{\partial s} d s= \\
-\frac{\partial p A}{\partial s} d s+p \frac{\partial A}{\partial s} d s=-\frac{\partial p}{\partial s} A d s
\end{gathered}
$$

El componente longitudinal de la fuerza tangencial de resistencia a la fricción o viscosidad, se opone siempre al flujo, y por ello es negativo; se cuantifica en términos del esfuerzo tangencial medio $\tau_{0}$ en la sección y el perímetro de contacto $P$ entre líquido y pared. Dicha fuerza vale: $-\tau_{0} P d s$. La fuerza de cuerpo se debe al peso del VC, $g \rho A d s$, y su componente longitudinal es: $-g \rho A \operatorname{sen} \theta d s$; pero $\operatorname{sen} \theta=\partial z / \partial s$ , de manera que su magnitud es: $-g \rho A(\partial z / \partial s) d s$.
El intercambio neto de cantidad de movimiento entre el VC y el exterior resulta: $\frac{\partial \rho, Q \beta V}{\partial s} d s$, que se desarrolla como sigue:

$$
\frac{\partial \rho, Q \beta V}{\partial s} d s=\beta V \frac{\partial \rho Q}{\partial s} d s+\rho A V \frac{\partial \beta V}{\partial s} d s
$$

Pero: $\frac{\partial}{\partial s}\left(\beta V^{2}\right)=V \frac{\partial \beta V}{\partial s}+\beta V \frac{\partial V}{\partial s}$,

o bien: $\rho A V \frac{\partial \beta V}{\partial s}=\rho A \frac{\partial}{\partial s}\left(\beta V^{2}\right)-\rho A \beta V \frac{\partial V}{\partial s}$;

por tanto: $\frac{\partial \rho, Q \beta V}{\partial s} d s=\beta V \frac{\partial \rho Q}{\partial s} d s+\rho A \frac{\partial}{\partial s}\left(\beta V^{2}\right) d s-$

$$
\rho A \beta V \frac{\partial V}{\partial s} d s
$$

con $\rho d v=\rho A d s y d s$ independiente de $t$, la variación en el tiempo de la cantidad de movimiento del VC se convierte en

$$
\frac{\partial}{\partial t} \oiiint_{V C} v \rho d v=\frac{\partial}{\partial t}(\rho A V) d s=\rho A \frac{\partial V}{\partial t} d s+V \frac{\partial \rho A}{\partial t} d s
$$

Con los resultados anteriores y dividiendo entre

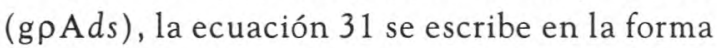

$$
\begin{gathered}
\frac{1}{g \rho} \frac{\partial p}{\partial s}+\frac{\tau_{0} P}{g \rho A}+\frac{\partial z}{\partial s}+\frac{\beta V}{g \rho A} \frac{\partial \rho Q}{\partial s}+\frac{1}{g} \frac{\partial}{\partial s}\left(\beta V^{2}\right)-\frac{\beta V}{g} \frac{\partial V}{\partial s}+\frac{1}{g} \frac{\partial V}{\partial t} \\
+\frac{V}{g \rho A} \frac{\partial \rho A}{\partial t}=0
\end{gathered}
$$

Algunos términos se pueden transformar como se muestra a continuación. Debido a que:

$$
\frac{\partial}{\partial s}\left(\frac{p}{g \rho}\right)=\frac{1}{g \rho} \frac{\partial p}{\partial s}-\frac{p}{g \rho} \frac{1}{\rho} \frac{\partial \rho}{\partial s}
$$

Resulta:

$$
\frac{1}{g \rho} \frac{\partial p}{\partial s}=\frac{\partial}{\partial s}\left(\frac{p}{g \rho}\right)+\frac{p}{g \rho} \frac{1}{\rho} \frac{\partial \rho}{\partial s}
$$

También:

$$
\frac{1}{g} \frac{\partial}{\partial s}\left(\beta V^{2}\right)=2 \frac{\partial}{\partial s}\left(\beta \frac{V^{2}}{2 g}\right)=\frac{\partial}{\partial s}\left(\beta \frac{V^{2}}{2 g}\right)+\frac{\beta V}{g} \frac{\partial V}{\partial s}+\frac{V^{2}}{2 g} \frac{\partial \beta}{\partial s}
$$


y de la ecuación de continuidad (1a):

$$
\frac{\partial \rho Q}{\partial s}=-\frac{\partial \rho A}{\partial t}=-\left(\rho \frac{\partial A}{\partial t}+A \frac{\partial \rho}{\partial t}\right)
$$

Sustituyendo las tres últimas expresiones en la ecuación 32 , al reordenar los términos se obtiene

$$
\begin{gathered}
\frac{\partial z}{\partial s}+\frac{\partial}{\partial s}\left(\frac{p}{g \rho}\right)+\frac{\partial}{\partial s}\left(\beta \frac{V^{2}}{2 g}\right)+\frac{p}{g \rho} \frac{1}{\rho} \frac{\partial \rho}{\partial s}+\frac{V^{2}}{2 g} \frac{\partial \beta}{\partial s}-(\beta-1) \frac{V}{g} \\
\left(\frac{1}{A} \frac{\partial A}{\partial t}+\frac{1}{\rho} \frac{\partial \rho}{\partial t}\right)+\frac{1}{g} \frac{\partial V}{\partial t}+\frac{\tau_{0} P}{g \rho A}=0
\end{gathered}
$$

O bien, si se acepta que los cambios de $\rho$ son menos importantes que los restantes términos, es decir, $(1 / \mathrm{A})(\partial \mathrm{A} / \partial \mathrm{t})>>1 / \rho(\partial \rho / \partial \mathrm{t})$, se obtiene

$$
\begin{gathered}
\frac{\partial}{\partial s}\left(z+\frac{P}{g \rho}+\beta \frac{V^{2}}{2 g}\right)+\frac{V^{2}}{2 g} \frac{\partial \beta}{\partial s}-2(\beta-1) \frac{V^{2}}{2 g} \frac{1}{Q} \frac{\partial A}{\partial t} \\
+\frac{1}{g} \frac{\partial V}{\partial t}+\frac{\tau_{0} P}{g \rho A}=0
\end{gathered}
$$

Esta es la ecuación diferencial de cantidad de movimiento más general para un flujo unidimensional no permanente de un líquido. Cuando se compara con la ecuación diferencial de la energía 26 para un flujo de la misma condición, se encuentran diferencias que pueden ser grandes o pequeñas según el conducto de que se trate. En efecto, ambas son aplicables a conductos a presión y a canales, pero en cada caso, existen diferentes formas según el tipo de flujo al que se aplique, como se presenta a continuación.

a) Para encontrar equivalencia en la irreversibilidad, ésta se tendría que explicar en la ecuación de la cantidad de movimiento como la energía consumida por el trabajo que tienen que realizar las fuerzas de fricción que se oponen al flujo, es decir, representan la pendiente de la línea de fricción en la forma

$$
\frac{d h_{f}}{d s}=S_{f}=\frac{\tau_{0} P}{g \rho A}
$$

a diferencia de la ecuación 25 donde la irreversibilidad es la pendiente de la línea de energía en la ecuación 26.

Ambas pendientes serían iguales sólo si A no cambia a lo largo de $s$, es decir, $\partial A / \partial s=0$, y la pérdida de fricción es la única que interviene. El trabajo que las fuerzas tangenciales efectúan en el contorno del VC se anula en la ecuación de energía por otras razones. Por tanto la ecuación 33 sería ahora

$$
\begin{gathered}
\frac{\partial}{\partial s}\left(z+\frac{p}{g \rho}+\beta \frac{V^{2}}{2 g}\right)+\frac{V^{2}}{2 g} \frac{\partial \beta}{\partial s}-2(\beta-1) \frac{V^{2}}{2 g} \frac{1}{Q} \frac{\partial \mathrm{A}}{\partial t} \\
+\frac{1}{g} \frac{\partial V}{\partial t}+\frac{d h_{f}}{d s}=0
\end{gathered}
$$

b) Cuando se asume que $\partial \beta / \partial s=0$, la suposición sería más aceptable en un conducto a presión que en un canal y en éste, bastante menos si es de sección compuesta (muy irregular). La ecuación 35 sería entonces

$$
\frac{\partial}{\partial s}\left(z+\frac{p}{g \rho}+\beta \frac{V^{2}}{2 g}\right)-2(\beta-1) \frac{V^{2}}{2 g} \frac{1}{Q} \frac{\partial A}{\partial t}+\frac{1}{g} \frac{\partial V}{\partial t}+\frac{d h_{f}}{d s}=0
$$

que es diferente de la ecuación diferencial de la energía (26).

c) Cuando sólo se acepta que $\alpha=\beta=1$, la ecuación 35 resulta

$$
\frac{\partial}{\partial s}\left(z+\frac{p}{g \rho}+\frac{V^{2}}{2 g}\right)+\frac{1}{g} \frac{\partial V}{\partial t}+\frac{d h_{f}}{d s}=0
$$

que es diferente de su similar 27 de la energía.

Para el caso del golpe de ariete, la ecuación 37 se puede escribir en función de la carga piezométrica $H$ y expresar la pendiente de fricción $d h_{f} / d s=S_{f}=f V^{2} / 2 g D$, donde $f$ es el factor de fricción de Darcy Weisbach.

$$
\frac{\partial}{\partial s}\left(H+\frac{V^{2}}{2 g}\right)+\frac{1}{g} \frac{\partial V}{\partial t}+\frac{f V|V|}{2 g D}=0
$$

Como la fricción se opone al movimiento, $V^{2}$ se expresa como $V|V|$ a fin de considerar el sentido del flujo; al multiplicar por g la ecuación 38a, y desarrollar $\partial \mathrm{V}^{2} / \partial s=2 \mathrm{~V}(\partial \mathrm{V} / \partial s)$, dicha ecuación se puede expresar como sigue

$$
g \frac{\partial H}{\partial s}+V \frac{\partial V}{\partial s}+\frac{\partial V}{\partial t}+\frac{f V|V|}{2 D}=0
$$

que es la ecuación dinámica para un líquido compresible en una tubería elástica.

d) Cuando el flujo es no permanente, pero en un conducto cerrado rígido sin cambios violentos de la presión (oscilaciones en masa) $\partial A / \partial t=0, y \partial \beta / \partial s=0$; la ecuación 35 se convierte en 


$$
\frac{\partial}{\partial s}\left(z+\frac{p}{g \rho}+\beta \frac{V^{2}}{2 g}\right)+\frac{1}{g} \frac{\partial V}{\partial t}+\frac{d h_{f}}{d s}=0
$$

que es diferente a su similar 28 de la energía, a menos que se haga $\alpha=\beta=1$, en cuyo caso se convierte en

$$
\frac{\partial}{\partial s}\left(z+\frac{p}{g \rho}+\frac{V^{2}}{2 g}\right)+\frac{1}{g} \frac{\partial V}{\partial t}+\frac{d h_{f}}{d s}=0
$$

idéntica a la que resultaría de la ecuación 28 , con la única diferencia en la pérdida $\left(h_{r}\right.$ por $\left.h_{f}\right)$.

Si se trata de un canal, vale la ecuación 35 ya que no son aceptables las suposiciones.

Un caso particular es su aplicación a una cámara de oscilación, en el cual se considera que la carga de velocidad en el conducto y en la cámara de oscilación son despreciables comparadas con las de presión que se generan en los mismos. Bajo esta consideración, la ecuación 39b queda

$$
\frac{\partial}{\partial s}\left(z+\frac{p}{g \rho}\right)+\frac{1}{g} \frac{\partial V}{\partial t}+\frac{d h_{f}}{d s}=0
$$

con referencia a la figura 3 , al integrar la ecuación 40 desde el almacenamiento hasta la cámara, es decir, a lo largo de la longitud $L$, se tiene

$$
z+\frac{L}{g} \frac{d V}{d t}+h_{f}=0
$$

e) Cuando el flujo es permanente, se anulan los términos que dependen del tiempo, $d h_{f} / d s=\partial h_{f} / \partial s$, y la ecuación 35 sería

$$
\frac{\partial}{\partial s}\left(z+\frac{p}{g \rho}+\beta \frac{V^{2}}{2 g}+h_{f}\right)+\frac{V^{2}}{2 g} \frac{\partial \beta}{\partial s}=0
$$

distinta de su similar, la 29 de la energía.

Si se trata de un conducto a presión se puede aceptar que, $\partial \beta / \partial s=0$, y se obtiene

$$
\frac{\partial}{\partial s}\left(z+\frac{p}{g \rho}+\beta \frac{V^{2}}{2 g}+h_{f}\right)=0
$$

que tampoco coincide con la propia ecuación 29 de la energía, a menos que en ambas se acepte que $\alpha=\beta=1$ y que $h_{r}=h_{f}=0$, en cuyo caso, se llega a la ecuación de Bernoulli, para la línea de corriente coincidente con el eje.

\section{Referencia}

Sotelo G. y Cafaggi A. (1998). Ecuaciones diferenciales del flujo unidimensional, en: Memorias del XVIII Congreso Latinoamericano de Hidráulica. Oaxaca. México.

\section{Semblanza de los autores}

Gilberto Sotelo-Ávila. Es ingeniero civil, maestro en ingeniería y doctor en ingeniería por parte de la Facultad de Ingeniería UNAM. Inició su actividad universitaria en 1958 como investigador en el Instituto de Ingeniería, profesor en licenciatura y posgrado. Recibió el premio Universidad Nacional 1998, Docencia en Ciencias Exactas, así también el Premio Nacional Miguel A. Urquijo 1999, otorgado por el Colegio de Ingenieros Civiles de México al mejor artículo técnico publicado durante 1997 y 1998. Participó en la planeación y supervisión de aproximadamente 250 obras de ingeniería civil dentro y fuera del país.

Adriana Cafaggi-Félix. Ingeniera Civil egresada en 1980 de la Facultad de Ingeniería UNAM, obtuvo el grado de maestra en ingeniería hidráulica en 1985 en la misma institución. Fue jefa de laboratorio y del Departamento de Hidráulica y actualmente es profesora de carrera en el Departamento de Ingeniería Hidráulica. Ha participado en congresos nacionales e internacionales y es autora de varios artículos e instructivos de prácticas para el Laboratorio de Hidráulica. 